Service social

\title{
Social Work with Groups in Health Settings, par Abraham Lurie, Gary Rosenberg et Sidney Pinsky (éd.), Prodist, New York, 1982, 124 pages.
}

\section{Lise Tessier}

Volume 39, numéro 1, 1990

Le groupe ici et ailleurs

URI : https://id.erudit.org/iderudit/706473ar

DOI : https://doi.org/10.7202/706473ar

Aller au sommaire du numéro

Éditeur(s)

École de service social de l'Université Laval

ISSN

1708-1734 (numérique)

Découvrir la revue

Citer ce compte rendu

Tessier, L. (1990). Compte rendu de [Social Work with Groups in Health Settings, par Abraham Lurie, Gary Rosenberg et Sidney Pinsky (éd.), Prodist, New York, 1982, 124 pages.] Service social, 39(1), 200-201. https://doi.org/10.7202/706473ar d'utilisation que vous pouvez consulter en ligne.

https://apropos.erudit.org/fr/usagers/politique-dutilisation/ 
traités mais aussi à cause des types de contenus qu'on y retrouve : certains articles présentent des notions davantage théoriques, souvent appuyées par des résultats de recherche, d'autres décrivent et discutent d'expériences concrètes d'intervention. De plus, certains se situent davantage dans une perspective thérapeutique alors que d'autres sont nettement orientés vers le soutien social et le développement communautaire.

Lise Darveau-Fournier

École de service social

Université Laval

\begin{abstract}
Note
1 M.A. Straus (1979). "Measuring intrafamily conflict in violence : The conflict tactics (CT) scales », Journal of marriage and family, vol. $41: 75-78$.
\end{abstract}

\title{
Social Work with Groups in Health Settings, par Abraham LURIE, Gary ROSENBERG et Sidney PINSKY (éd.), Prodist, New York, 1982, 124 pages.
}

Ce livre fait suite à un colloque sur l'utilisation des groupes en milieu de santé, qui fut organisé conjointement par les Départements de service social du Long Island Jewish Hillside Medical Center et du Queens Hospital Center de New York.

L'idée de ce symposium est née de la constatation que l'approche de groupe était de plus en plus utilisée en milieu de santé et qu'on lui reconnaissait des valeurs à la fois thérapeutiques et économiques. On reconnaît aussi que les changements majeurs survenus dans le champ de la santé, tels l'augmentation des clientèles ayant des maladies chroniques, l'accent mis sur les droits des bénéficiaires, les coûts très élevés des soins de santé, les enjeux liés à la qualité des soins et à la responsabilisation, et une réduction dans la durée des hospitalisations avec en corollaire un accent sur la planification du congé, sont tous des défis qui obligent à identifier les enjeux et les problèmes qui se posent si on veut développer des services de groupe.

Les participants à la rédaction de ce livre sont connus pour leur contribution théorique au développement du service social des groupes (Alex Gitterman et Beulah Rothman) ou sont des professionnels chevronnés du milieu de la santé (Abraham Lurie, Gary Rosenberg et Sidney Pinsky, éditeurs de l'ouvrage). La douzaine d'articles de ce livre couvre le contenu suivant :

- une partie plus théorique où Alex Gitterman souligne les avantages et les problèmes à développer des approches de groupe en milieu de santé, et touche à la formation, aux buts et à la composition d'un groupe, aux facteurs temps, espace et taille du groupe, de même qu'aux problèmes de recrutement en milieu institutionnel. Beulah Rothman va plus loin en mettant l'accent sur les principes dans l'utilisation du groupe qui favorisent la santé, et sur l'importance de la structure du groupe et du contenu de la vie de groupe. Une application de ces principes est présentée par Elaine Lonergan. 
- une recension des publications, faite par Gary Rosenberg et Grace Neill, met en évidence la pauvreté des données objectives sur les expériences de groupe en cours et l'absence d'évaluation de résultats. Selon eux, la documentation ne rend pas justice à la valeur de l'intervention comme telle. IIs pensent que le processus de groupe est une méthode efficace tant pour les parents et les familles que pour le personnel, et qu'elle peut être utilisée pour répondre à un large éventail d'objectifs : donner de l'information, faire de l'éducation, amener des changements d'ordre thérapeutique, aider à la résolution de problèmes, avoir des activités de support et de prévention pour les patients et les familles.

- mis à part l'article de Tuzman qui décrit les éléments d'une relation de travail entre les systèmes formels de distribution de services en milieu de santé et les conseils d'administration des groupes d'entraide, les autres articles sont des illustrations de pratiques de groupes : patients avec problèmes de langage et leurs conjoints (Rosenfeld), préparation à la sortie de patients d'un service d'orthopédie, et communauté thérapeutique pour les consommateurs d'alcool à problèmes (Dougherty), facteurs socioculturels qui empêchent le développement de l'ego (Haber), modifications du comportement (Ron), thérapie sexuelle (Adelson et Peress), hospitalisations en psychiatrie (Halpern et Dlugacz).

Ce livre a le mérite d'ouvrir la discussion sur la place du service social des groupes en milieu de santé. Certains périodiques comme Social Work with Groups, Health and Social Work et la revue Social Work publient à l'occasion un article sur une expérience de groupe en milieu de santé, mais c'est la première fois, à ma connaissance, qu'on essaie de faire le point sur le sujet en combinant concepts théoriques sur le groupe, documentation sur l'évaluation de l'intervention et descriptions d'expériences pratiques en milieu de santé. Ceci permet de mesurer tout le chemin qu'il reste à parcourir. Un des obstacles saute aux yeux en examinant la recension des écrits (p. 41) : le nombre de spécialités médicales et la dispersion des intervenants sociaux à l'intérieur de ces spécialités. 